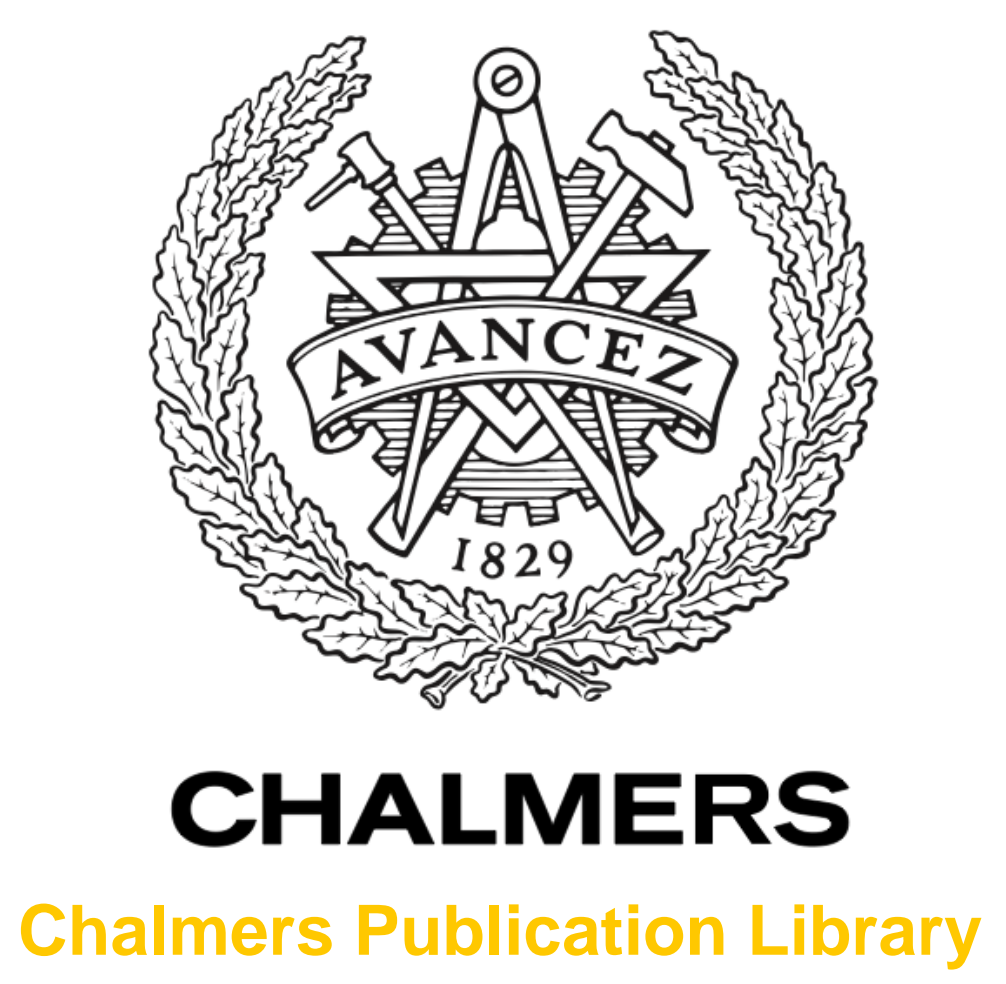

\title{
Software-Defined Radio Direct Correlation GNSS Reflectometry by Means of GLONASS
}

This document has been downloaded from Chalmers Publication Library (CPL). It is the author's version of a work that was accepted for publication in:

IEEE Journal of Selected Topics in Applied Earth Observations and Remote Sensing (ISSN: 1939-1404)

Citation for the published paper:

Hobiger, T. ; Haas, R. ; Löfgren, J. (2016) "Software-Defined Radio Direct Correlation GNSS Reflectometry by Means of GLONASS". IEEE Journal of Selected Topics in Applied Earth Observations and Remote Sensing, vol. 9(10), pp. 4834-4842.

Downloaded from: http://publications.lib.chalmers.se/publication/244214

Notice: Changes introduced as a result of publishing processes such as copy-editing and formatting may not be reflected in this document. For a definitive version of this work, please refer to the published source. Please note that access to the published version might require a subscription. 


\title{
Software defined radio direct correlation GNSS reflectometry by means of GLONASS
}

\author{
Thomas Hobiger, Rüdiger Haas and Johan Löfgren
}

\begin{abstract}
Ground-based GNSS reflectometry systems can be realized by different means. The concept of correlation between direct and reflected GNSS signals is basically possible with all GNSS systems. However, using signals from the Russian GLONASS system simplifies the signal processing so that software defined radio components can be used at replace expensive hardware solutions. This paper discusses how such a solution, called GLONASS-R, can be realized by using entirely off-the-shelf components. Field tests with such a system demonstrate the capability to monitor sea surface heights with a precision of $3 \mathrm{~cm}$ or better even with a sampling rate of $1.5 \mathrm{~Hz}$. The flexibility of a software defined radio and the simple concept of GLONASS-R allows to build such a system with low costs and adapt it to the needs of any ground-based GNSS-R problem.
\end{abstract}

\section{Index Terms}

GNSS-R, GLONASS, software defined radio, GPU, sea-level

\section{INTRODUCTION}

In order to monitor sea-level changes it is not only necessary that precise measurements are carried out over long time, but it is also crucial that these measurements are geo-referenced in an absolute global reference frame [1]. In particular, when data collected with coastal sea-level sensors (hereafter called "tide gauges") are interpreted, it is important to know the movements of the continental crust at the sites where these sensors are deployed. Under this aspect, ground based GNSS reflectometry (GNSS-R) appears to be an attractive application for monitoring long-term sea-level changes since GNSS-R relates directly to a global reference frame.

As for ground-based GNSS-R one can distinguish between two different concepts. First, there are systems which receive direct and reflected signals via one single antenna and require the extraction of reflector heights from the interpretation of signal-to-noise ratio (SNR) data (see e.g. [2]-[6]). In these cases usually commercial geodetic GNSS equipment is used to obtain information about reflector heights. As a second method of retrieving groundbased sea-level measurements one can utilize two antennas, one up-looking antenna for receiving the direct signal from the satellites and one down-looking antenna which is dedicated to receiving reflected signals from the water surface (cf. [7]-[10]). Also for this method commercial GNSS equipment is used, however sea-level heights are

T. Hobiger, R. Haas and J. Löfgren are with the Department of Radio and Space Science,Chalmers University of Technology, SE-412 96, Gothenburg, Sweden e-mail: thomas.hobiger@chalmers.se.

Manuscript received Month XX, 2014; revised Month YY, 2015. 
obtained from analysing carrier-phase observations of the direct- and reflected signals. In general, it can be stated that single (up-looking) antenna studies are usually carried out when existing ground-based GNSS infrastructure has already been deployed and its data can be easily accessed. Concepts with two antennas are usually developed when reflected signals need to be processed by dedicated algorithms and/or when the scattered power is low and only high-gain downward looking antennas can help to detect ground reflections.

In the following, the dual-antenna concept will be chosen and a simple direct correlation reflectometry system by means of GLONASS and software-defined radio will be presented.

\section{GLONASS-R}

In its most simple form, GNSS-R operates like a passive bistatic radar [11], i.e. processing reflections from objects or surfaces by taking advantage of natural or artificial signal transmitters. Such a concept is realized by comparing direct and reflected signals concerning delay and signal strength. In doing so, one can derive properties of the reflector and its location w.r.t. the receiving antennas [12]. Since most GNSS signals are based on a code division multiple access (CDMA) scheme, correlation of direct and reflected signals requires complicated signal processing methods as signals from several satellites appear in the same frequency band. Among those approaches, satellite selection via antenna directivity [13] is the most frequently used method to enable direct correlation of the signals. However, this requires that the beam of at least one antenna is steerable and/or limited in its spatial extent in order to suppress signals from other GNSS satellites.

An alternative solution that enables direct correlation has been presented by [14] where the authors utilized the frequency division multiple access (FDMA) encoding scheme of the Russian Global Navigation Satellite System (GLONASS) space segment. Results from this first study indicate that sea surface height can be monitored in real-time with $\mathrm{cm}-\mathrm{level}$ precision from ground-based GNSS-R installations. However, the need for complex and expensive RF front-ends. down-conversion stages and A/D converters made the so-called GLONASS-R system rather unattractive for being duplicated at other coastal sites. This problem is thought to be overcome by replacing those unflexible components with off-the-shelf software-defined radio equipment. This does not only lead to a drastic price reduction but also increases the flexibility of the GLONASS-R concept. Furthermore, advances in graphics processing unit (GPU) architecture enable us now to change the signal processing chain in a way which handles the data more efficiently, thus leading to a higher throughput while still preserving the real-time capability of the original concept.

\section{A. Software-defined radio integration}

Software-defined radio (SDR) [15] is a very powerful and flexible concept for prototyping and quick realization of projects without the need of application-specific integrated circuit (ASIC) boards. Thus, SDR is a very appealing solution which helps to implement a novel GNSS-R concept with much lower prototyping and development cost. As signal processing can be carried out on a PC, such a GNSS-R solution can be built with off-the-shelf components and adapted in a very flexible way before or during any development phase. Therefore, also [14] have chosen SDR in order to implement their concept of GLONASS-R. However, their first implementation was limited in flexibility 
due to the fixed frequency setting of the RF front-ends and the choice of the analogue-digital (A/D) converters. In order to overcome these limitations, the Ettus URSP N210 has been selected to increase the flexibility of the receiver. It was demonstrated in [16] that the USRP N210, which covers all GNSS frequencies, can be used to realize a GNSS software receiver. Thus, this technology was chosen in order to develop a new version of the GLONASS-R system. Figure 1 depicts how two USRPs are used to acquire continuous data-streams of digitized GLONASS signals.

In order to make sure that direct, i.e. right-hand circular polarized (RHCP), and reflected, i.e. left-hand polarized (LHCP), signals are received coherently, a so-called MIMO cable is applied between the two USRPs. Although only coherence between the two units is required for an interferometric system, external $10 \mathrm{MHz}$ and 1 pulse-per-per second (PPS) signals are fed to the "master" device. In doing so, one can, at any stage, independently process either RHCP or LHCP data-streams and is not affected by drifts of the USRP on-board oscillators. The MIMO cable simplifies also data-transfer as samples taken from the "slave" device are sent to the "master" unit from where the data are time-stamped, packetized and transmitted over a standard 1 Gigabit Ethernet (GbE) connection. The latter feature allows to put the GLONASS-R system at remote places or harsh environment, given that a stable GbE connection between the "master" USRP and the PC, which carries out the signal processing, is provided.

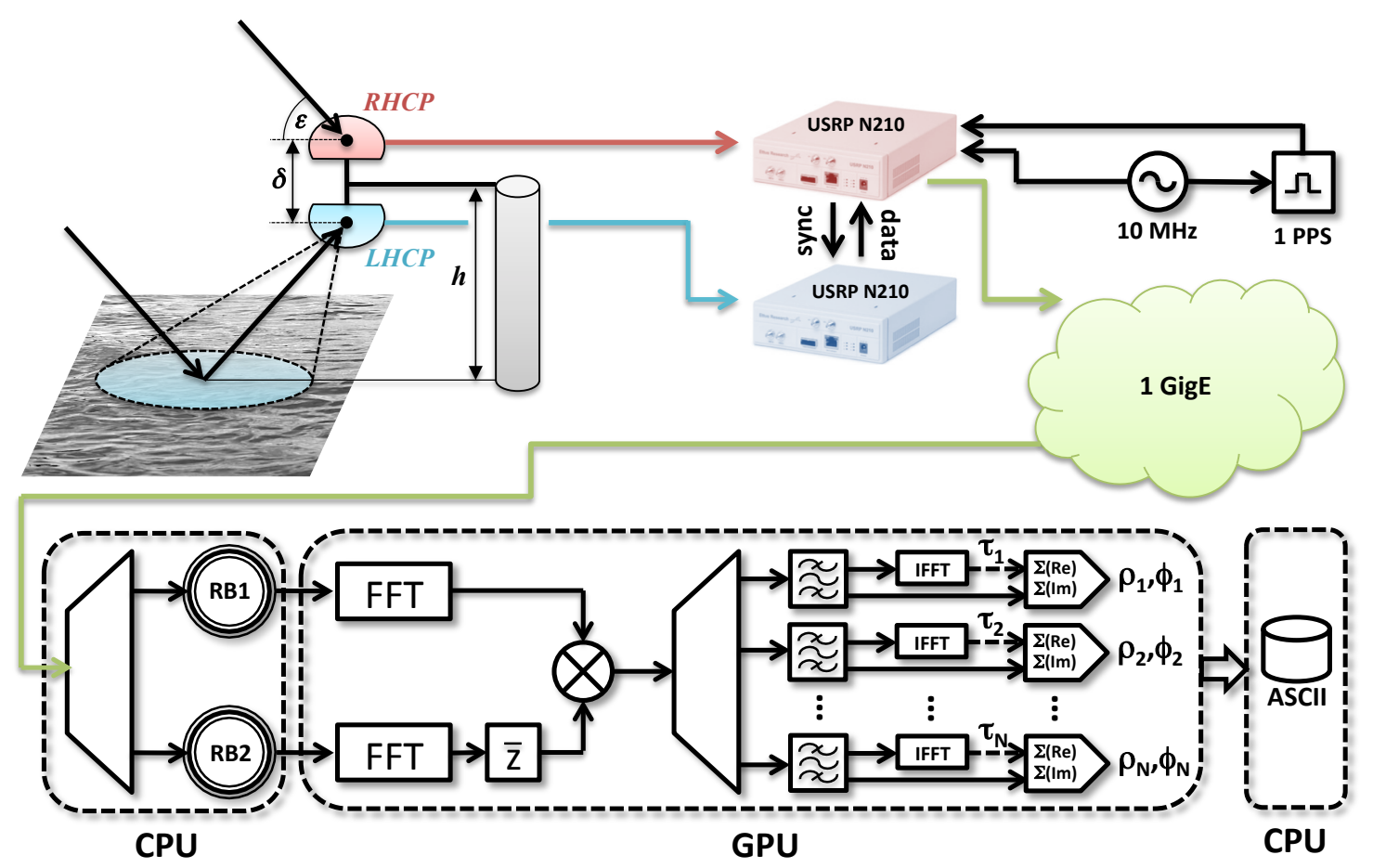

Fig. 1. Overview of the GLONASS-R concept. Direct and reflected signals are received via dedicated RHCP and LHCP antennas and digitized by two URSP devices which are synchronized with each other. The sampled data are sent to a commodity PC over a 1 GbE connection and stored in two circular buffers. A thread which controls the processing on the GPU fetches data from those buffers, copies them to the GPU and computes interferometric delays, phases and amplitudes for all visible GLONASS satellites. Once the processing of a data batch has been completed, results are copied back to the CPU and the next sampling data are copied to the GPU. 
As the sampling rate of the IQ digital down converter (DDC) equals the receiver's bandwidth 12.5 Mega samples per second (Msps) are sufficient to cover the whole GLONASS L1 band in case the center frequency is set to $1602 \mathrm{MHz}$. Given that the real and imaginary parts of a sample are represented as 8 bit words each, one gets a total data-rate of $2 \times 2 \times 8$ bit $\times 12.5 \cdot 10^{6}=400$ Mbps that contains both RHCP and LHCP data-streams. Such a data-rate is well below the GbE limit, though it poses the risk of back-logging when the signal processing chain cannot keep the pace with the incoming amount of data. In order to avoid bottlenecks and ensure real-time processing of the incoming data-stream, data buffering and signal processing have been split between the PC's central processing unit (CPU) and the graphic processing unit (GPU) which does the heavy signal processing. As already discussed in [14], data parallelism and the use of high-performance signal processing algorithms on the GPU ensure that even higher data-rates can be handled in real-time.

\section{$B$. The GLONASS-R receiver on the GPU}

A continuously running process on the CPU receives data packages from the USRPs and stores them in circular buffers. A second process runs a program on the GPU which picks data from these buffers, and computes interferometric information between the direct and reflected signals. Other than GPS or similar CDMA based satellite systems, GLONASS signals enable direct correlation since the individual satellite signals are clearly distinguishable in the frequency domain. Thus, at its first stage the GPU-based receiver performs Fourier transforms on the RHCP and LHCP signals. In the original concept presented in [14] data-lengths of one millisecond were handled in this way. However, at a later stage coherent integration was required in order to achieve sufficient SNR and extract meaningful interferometric phases. This drawback has now been avoided by Fourier transforming 8388608 samples, which equals data from about 0.67 seconds, and making use of high-performance in-place Fast-Fourier Transformations (FFTs). NVIDIA's Compute Unified Device Architecture (CUDA) provides a library which also supports such long FFT transformation while still fulfilling the requirements for real-time processing of the incoming data-streams.

After RHCP and LHCP data-streams have been Fourier transformed (cf. Fig. 1) and the complex cross-spectrum has been obtained, simple orbital information in the form of two-line element [17] strings and a rough estimate of the receiver location are sufficient in order to determine which satellites are above the local horizon and need to be considered further in the signal processing chain. Based on the knowledge of which GLONASS satellite is visible at the site, a bandpass filter with a width of $562.5 \mathrm{kHz}$ and a center frequency corresponding to the carrier frequency of that satellite can be applied in order to avoid that the interferometer phase contains information other than those from the selected satellite. As depicted in Fig. 1, an inverse Fourier transformation immediately provides the interferometric delay between the direct and reflected signal. By using this coarse delay information, one can rotate the cross-spectral phases across the whole band-pass, provide a delay corrected complex cross-spectrum and obtain interferometric phase and amplitude for a particular satellite. This approach needs only 2 FFTs and $S$ inverse FFTs in order to obtain the interferometric information for all $S$ satellites for a given data batch. Memory restrictions on the GPU make it necessary that satellites are dealt with serially. In addition to the high-performance FFTs provided via CUDA, also fast algorithms for detection of the correlation peak, i.e. extraction of the interferometric delay, and the summation of the corrected cross-spectra are necessary. Both reduction tasks can be realized by taking 
advantage of the concept of parallel prefix computation [18], which maps perfectly to GPU architectures. In doing so, peak search and summation algorithms follow only $O\left(\log _{2}(N)\right)$ which makes the choice of large FFTs (of size $N$ ) even more attractive.

Performance tests with a NVIDIA Tesla K40 have shown that it is possible to run a complete GLONASS-R receiver with up to 12 satellites in real-time while maintaining data rates of 12.5 Msps from RHCP and LHCP channels.

After all visible satellites are processed, the GPU process transfers interferometric delays $\tau_{i}(t)$, phases $\phi_{i}(t)$, and correlation amplitudes $\rho_{i}(t)$ back to the CPU where this information is stored in text files. The GPU thread will then fetch the next batch of data from the ring-buffer and continue with the next data batch. This process is running continuously until the user stops either the CPU thread which controls the ring buffering or the thread which runs the GPU kernels. Based on this concept, successful and uninterrupted real-time operation of the GLONASS-R was confirmed over a period of several weeks (cf. Sec. III).

\section{Post-processing}

From the three observables, $\tau_{i}(t), \rho_{i}(t)$ and $\phi_{i}(t)$, the latter one, i.e. interferometric phase, is providing the most precise measurements which contain information about the geometric relation between the height $h(t)$ of the LHCP antenna above the reflector and the elevation angle $\varepsilon_{i}(t)$ under which the $i$-th satellite is seen. As the vertical distance $\delta$ between the two antenna phase centers is known with mm-accuracy one can express the observed phase as

$$
\phi_{i}(t)=\bmod _{2 \pi} \pi\left[2 h^{\prime}(t) \cdot \sin \varepsilon_{i}(t)\right]+\Delta \phi_{i}
$$

where $h^{\prime}(t)=h(t)+\frac{\delta}{2}$ has been introduced as the virtual height above the reflector. In Eq.1, $\bmod _{2 \pi}$ denotes the modulo $2 \pi$ operator. The unknown phase bias $\Delta \phi_{i}$ is caused by different electrical path lengths of the RHCP and LHCP signals, which have been confirmed to be constant over the duration of a satellite passage. Given that usually several GLONASS satellite are visible at the same time, Eq. 1 portrays an over-determined problem in the case that the functional model of the reflector height $h^{\prime}(t)$ is not expressed by more parameters than the total number of phase observations. Phases from seyeral satellites are obtained every 0.67 seconds and it is obvious that even a moderate choice for the temporal resolution of the reflector height leads to a strong over-determined problem. However, the inversion, i.e. the estimation of such a functional model in dependence of the observations, is not a trivial task due to the presence of the modulo operator. Classical least-squares approaches [19] cannot handle this problem due to the discontinuity caused by the modulo operator. Non-linear minimization algorithms could be applicable, however a-priori values for the unknown model parameters need to be chosen carefully in order to make sure that such an algorithm converges towards a global minimum. On the other side, if phases would be connected or unwrapped before Eq. 1 is inverted, the modulo operator disappears and the unknown number of integer phase cycles gets absorbed into the phase offset $\Delta \phi_{i}$. This leads to the idea of step-wise inversion of phase data.

1) Phase unwrapping: Connection of phases, hereafter called "phase unwrapping" requires that the interferometer phase is not varying by more than a half-cycle between two consecutive epochs. Assuming that the reflector height is not changing during one satellite passage, phase changes for low elevation angles can be approximated to first 
order by $h^{\prime} \frac{d \varepsilon_{i}(t)}{d t}$. Given that the GNSS-R system is located only a few tens of meters above the reflecting surface and considering that interferometric data are taken with sampling rates of more than $0.2 \mathrm{~Hz}$ it is obvious that phase connection should be feasible for all GNSS wavelengths. Knowing that $\left(\frac{d \varepsilon_{i}(t)}{d t}\right)_{\max } \approx 1.4 \cdot 10^{-4} \mathrm{rad} / \mathrm{s}$ and considering an extreme height of $100 \mathrm{~m}$, interferometer phases should not change by more than $7 \mathrm{~cm}$ (or a third of a GNSS wavelength) over $5 \mathrm{~s}$, i.e. using a sampling rate of $0.2 \mathrm{~Hz}$ However, this does not consider the fact that individual interferometric phases have an uncertainty that is inverse proportional to the SNR of the cross-correlated signals. This means that phase connection algorithms should not only consider rapid changes of the geometric delay but also deal with the problem that phase observations are noisier at lower elevation angles. Many mathematical software packages provide functions that connect phases, though all implementations tested in this study did not consider the global smoothness of the resulting unwrapped phase time series. On the other side, if one has a good enough functional model that explains the relation between the observed phases and the physical source, simple rounding can be used to unwrap the phases. In order to apply this idea to the interferometric phases from the GLONASS-R system one needs to assume that the reflector height is not changing during the data period which is considered for phase connection. Following Eq. 1 we can define the cost function

$$
F\left(p_{1}, p_{2}, t\right)=\bmod _{2 \pi}\left[p_{1} \cdot \sin \varepsilon_{i}(t)\right]+p_{2},
$$

and search for a global optimal set of parameters $\left(p_{1}, p_{2}\right)$ which minimize the sum of the squared residuals over one satellite passage, i.e.

$$
\sum\left[\phi_{i}(t)-F\left(p_{1}, p_{2}, t\right)\right]^{2} \rightarrow \min _{p_{1}, p_{2}} .
$$

Based on the set of parameters $\left(p_{1}, p_{2}\right)$ one can then unwrap the raw interferometer phases by adding/subtracting integer numbers of cycles $M_{i}$ for each observation so that $\left|\phi_{i}(t)-F\left(p_{1}, p_{2}, t\right)-2 \pi M_{i}\right|<\pi$. Figure 2 depicts the robustness of this phase unwrapping concept.

Unwrapping the raw interferometer phases with a standard function of Octave [20] fails when two or more consecutive epochs contain noisy data, causing the unwrapped phase to jump by more than one cycle. As Octave's algorithm does only connect phases based on the differences between consecutive data points, any wrong unwrapping at an epoch of high phase noise will lead to steps (cf. Fig. 2, middle plot) which can be later mis-interpreted as sea surface changes. The boot-strapping algorithm however produces a smooth time-series of unwrapped interferometer phases as shown in the lower plot of Fig. 2. Periods of low SNR, i.e. high phase noise, do not affect the overall shape of the unwrapped phase time series, and thus will not influence the inversion of sea surface height. If the sea surface changes by more than a phase cycle, i.e. roughly $20 \mathrm{~cm}$, the cost function Eq. 2 needs to be extended by a time variational term that considers temporal variations of the reflector height during one satellite passage. Another way of dealing with such a situation would be the splitting of satellite passage in sub-arcs, e.g. a rising and a setting part, in order to stay within the validity range of a constant height model during each arc period.

2) Fitting a model for the reflector height changes: A comparison of Eqs. 1 and 2 reveals that $p_{1}=2 h^{\prime}$ which means that one could obtain sea-level heights directly from the phase unwrapping process described in the prior section. However, this goes along with the crude assumption that the reflector height does not change during the passage of one satellite. Valuable information concerning temporal changes of the reflector height are lost when 


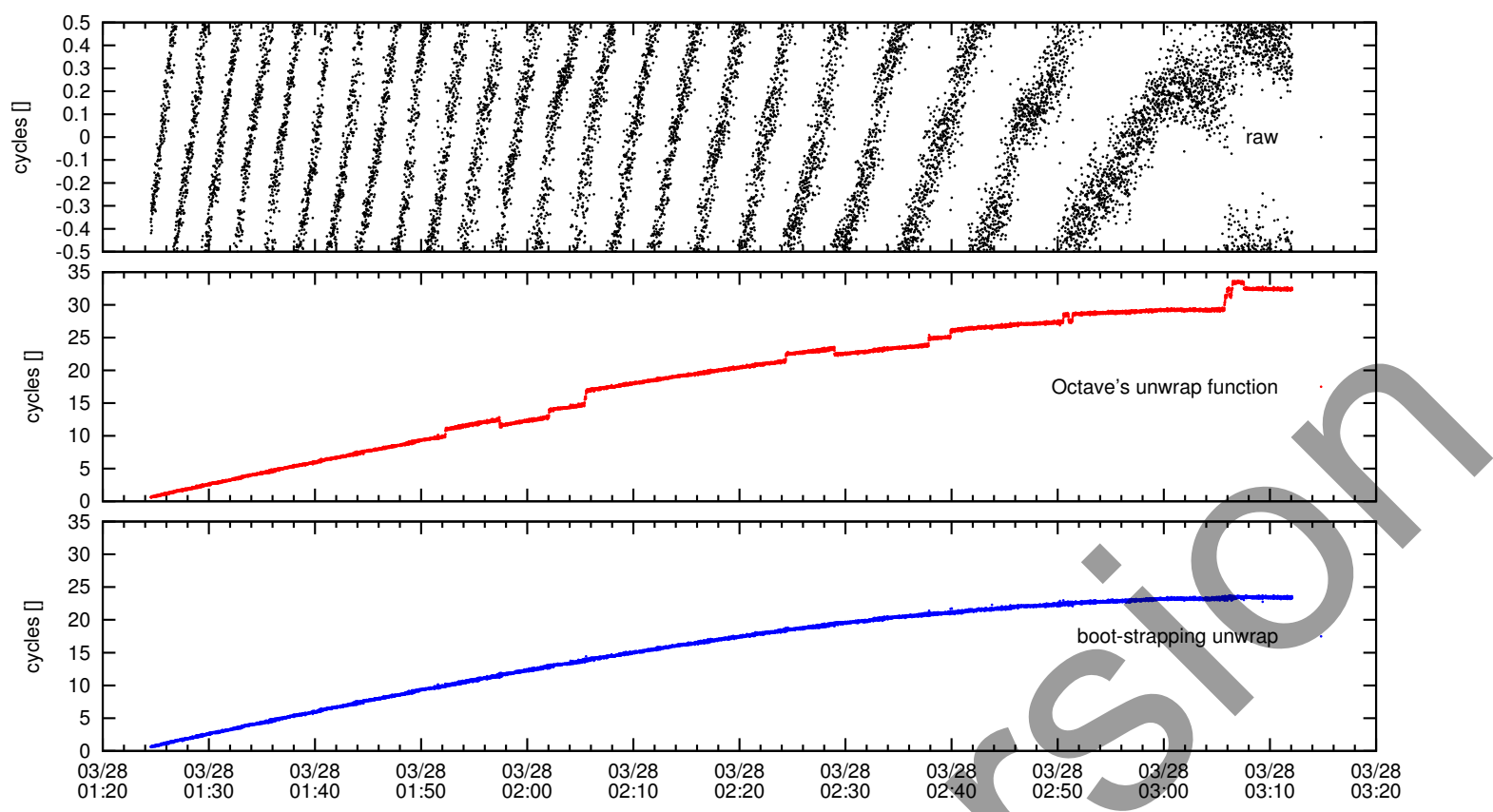

Fig. 2. Unwrapping interferometer phases from a test run in March 2015. The upper plot depicts the raw interferometer phases of a GLONASS satellite (Kosmos 2474) during its passage. Results from the unwrapping of these phases with the help of the Octave "unwrap" function are shown in the middle. It is clearly visible that phase noise at two or more consecutive epochs leads to discontinuities in the time series. The lower plot depicts the phase unwrapping results when a boot strapping method with a background model for the reflector height is applied (see Sec. II-C1). Using this approach all discontinuities are removed and the unwrapped phase time series can be used for reflector height retrievals (cf. Sec. II-C2 and II-C3).

using a constant height for each arc. This should be therefore avoided. Given that the unwrapped phase $\phi_{i}^{*}(t)$ has already be obtained, one can re-formulate Eq. 1 to

$$
\phi_{i}^{*}(t)=2 H^{\prime}\left(x_{0}, x_{1}, \ldots, x_{N}, t\right) \cdot \sin \varepsilon_{i}(t)+\Delta \phi_{i}
$$

where the temporal variation of the reflector height is approximated by an analytical function $H^{\prime}$ with $N$ parameters $x_{j}$. As described in [14], a quadratic B-spline $B_{j}^{2}(t)$ function is a suitable functional representation for temporal reflector height variations. The spacing of the knots relates to the temporal resolution of the model and determines the total number of unknowns which need to be estimated. This means that Eq. 4 can be expressed as a linear model in the form

$$
\phi_{i}^{*}(t)=2 \sin \varepsilon_{i}(t) \cdot \sum_{j=0}^{N} x_{j} N_{j}^{2}(t)+\Delta \phi_{i},
$$

where $x_{j}$ are the so called scaling coefficients for the corresponding quadratic B-spline functions with $N$ nodes. Given the high sampling rate of the raw data and the fact that always several satellites are tracked in parallel, one can determine the unknown coefficients $x_{j}$ by a simple weighted least-squares approach. In doing so, the interferometric correlation amplitudes $\rho_{i}$ can be taken as weights in order to avoid that phase data with low SNR contributes too much to the estimated model [14]. Phase offsets $\Delta \phi_{i}$ are estimated as an additional parameter for 
each arc and can be used for obtaining sea-level heights with a temporal resolution equal to the one of the raw interferometer phase.

3) High-rate sea surface heights: Given that phases are unwrapped and phase offsets $\Delta \phi_{i}$ have been estimated from the prior steps, one can easily invert Eq. 1 and obtains

$$
h^{\prime}(t)=\frac{\phi_{i}^{*}(t)-\Delta \phi_{i}}{2 \cdot \sin \varepsilon_{i}(t)} .
$$

This means that reflector heights are available with the same temporal resolution as the interferometer phases are output from the software receiver. However, as stated before usually more than one satellite is tracked per epoch. Thus it appears to be logical to average all the data at a given epoch in order to obtain the most robust estimate of reflector height at a particular point in time. It was suggested in [14] to compute a weighted average over all satellites at each epoch, i.e.

$$
h^{\prime}(t)=\frac{\sum_{t=t_{i}} \rho_{i}^{2} \frac{\phi_{i}^{*}(t)-\Delta \phi_{i}}{2 \sin \varepsilon_{i}(t)}}{\sum_{t=t_{i}} \rho_{i}^{2}} .
$$

In summary, GLONASS-R phase data can be inverted to sea level height in three steps, where in each step the temporal resolution of the obtained reflector height increases.

Step 1 Phase unwrapping algorithm and determination of arc-wise constant reflector heights (cf. Sec. II-C1),

Step 2 Low-pass filtered reflector height variations with a temporal resolution defined by the B-spline nodes (cf. Sec. II-C2),

Step 3 High-rate reflector heights using the arc phase biases from Step-2 (cf. Sec. II-C3).

\section{RESULTS}

In order to test the new GLONASS-R architecture and the post-processing strategies described above, a long-term test at the Onsala Space Observatory (OSO), Sweden was carried out in 2015. Figure 3 depicts the location of the GNSS-R antennas as well as the recently build tide gauge that is part of the Swedish network of tide gauges operated by the Swedish Meteorological and Hydrological Institute (SMHI). This test did not only aim at the evaluation of the system by comparison with other co-located sea-level instruments but also demonstrated that the software-defined radio concept can process the data in real-time over weeks without loosing a single epoch. As the GLONASS-R software receiver shares the same antennas with GNSS-R system [10] a direct comparison between the different systems is possible. The latter system is based on commercial GNSS receivers can provide singledifference solutions [10] when using tracking data of the up- and downward looking antenna/receiver pair. Both commercial receivers are tracking GPS and GLONASS on L1 and L2 each, four individual single-difference phase solutions can be obtained. Moreover, SNR data from the up-ward looking antenna/receiver can be inverted in order to obtain four additional independent solutions. Measurements from the SMHI tide gauge can be utilized in order to evaluate how well the independent GNSS-R solutions agree with this reference. Over a period of three weeks in 2015 all systems were operated in parallel and sea level height were measured with the individual instruments (cf. Fig. 4). 


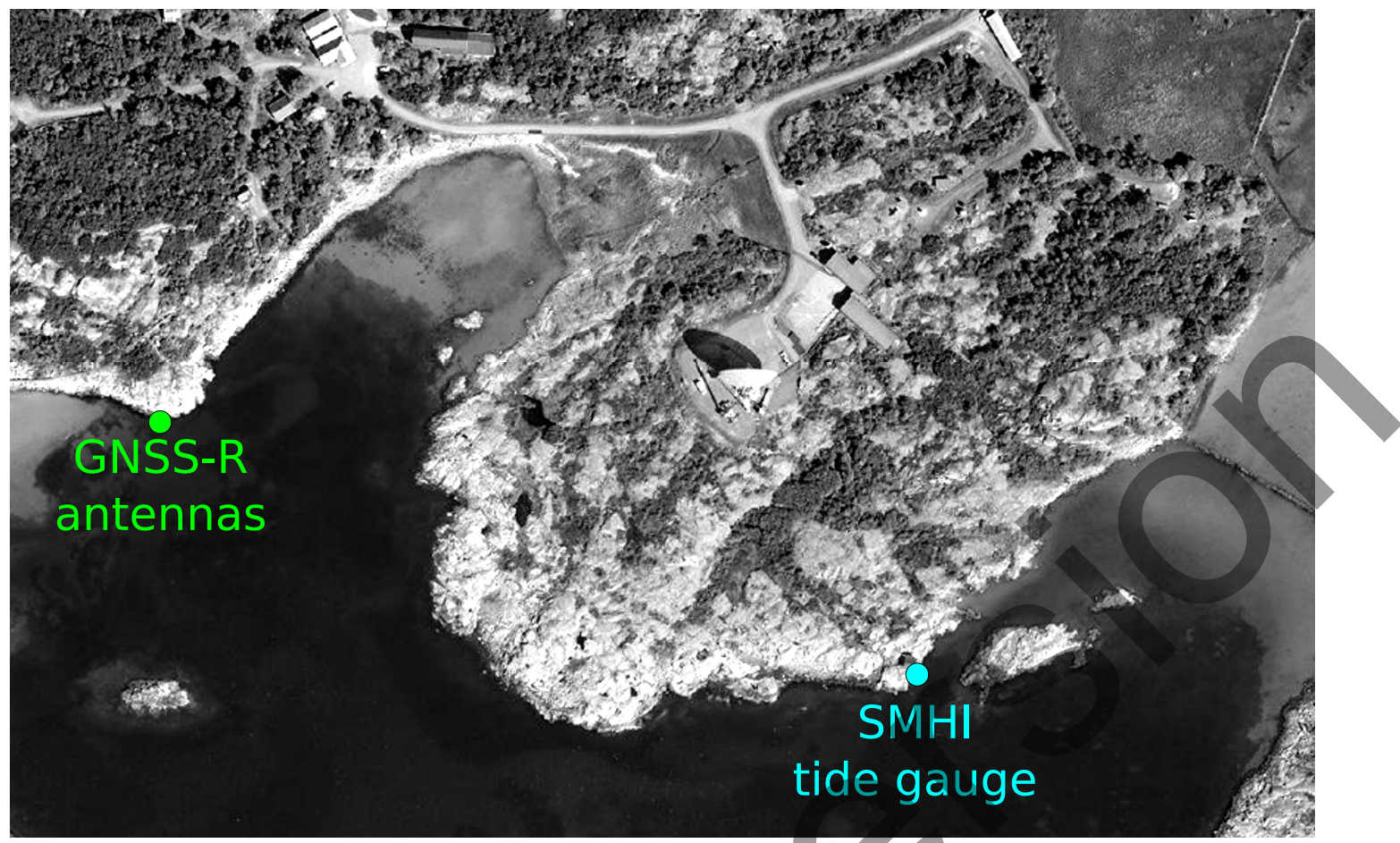

Fig. 3. Aerial image of the coastal area at the Onsala Space Observatory. The location of the GNSS-R system (latitude: 57.3930 deg, longitude: $11.9135 \mathrm{deg}$ ) in the western part is marked with a green bullet and the location of the newly built SMHI tide gauge (latitude: 57.3920 deg, longitude: $11.9190 \mathrm{deg}$ ) is depicted in cyan.

\section{A. Time series comparison}

Although all sensors were operated during the whole test period, clear gaps can be seen in some of the time series. First, the SMHI tide gauge underwent final testing before producing official measurements, including adjustment and calibration of the radar sensor and thus did not record data between June 15-19, 2015. The commercial GNSS receivers connected to the RHCP and LHCP antennas were recording during the whole period, but strong winds which lead to a rougher sea surface caused the receiver connected to the LHCP antenna to loose lock and thus made it impossible to apply the single-difference post-processing scheme during these periods. Therefore, four large gaps in the GNSS phase solutions can be found (cf. Fig. 4), leaving the SNR solutions as the only continuous time series over the whole period. However, the irregular and rather sparse temporal spacing of these time-series makes it impossible to deduce spectral characteristics from the SNR method.

The GLONASS-R system was operating all the time and recorded interferometer phases which were unwrapped with the method described in Sec. II-C1. The B-spline approach with a temporal resolution of two hour was used to low-pass filter these raw phases and estimate the phase biases for each satellite passage. By back substitution of these biases one can obtain sea-level measurements with a temporal resolution of about 0.67 seconds. As shown in Fig. 4 , the SMHI tide gauge data and the GLONASS-R measurements reveal the same pattern of sea level. Also the single difference GNSS phase solutions reveal a very similar pattern, though the large number of gaps make it difficult to judge this by naked eye. As for the SNR solutions the agreement is less clear as all of the four time 


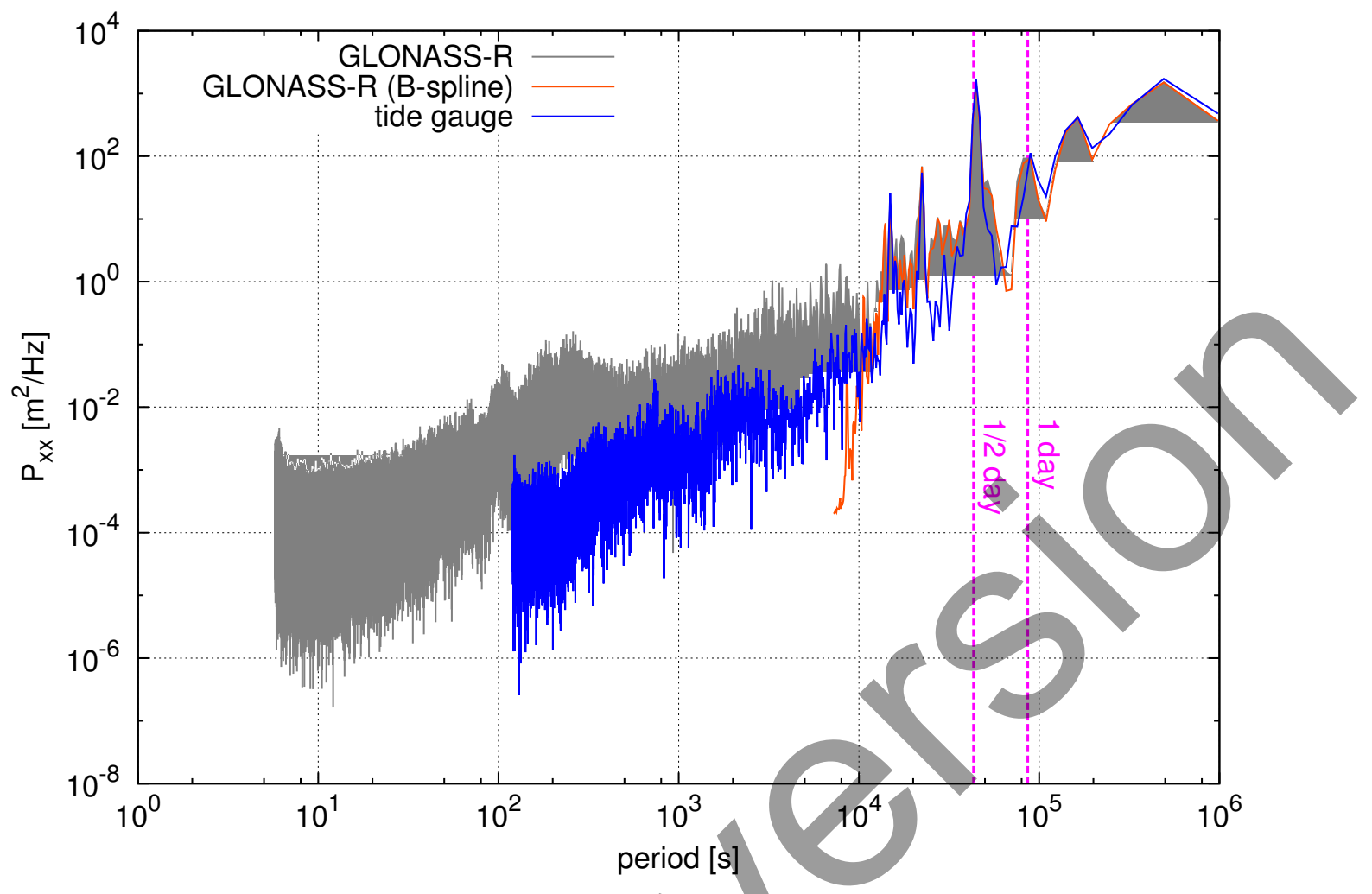

Fig. 4. Upper plot: Tide gauge measurements from 2015 are plotted together with GLONASS-R results and data obtained from the geodetic GNSS receivers. The latter include SNR and single difference solutions for GPS and GLONASS on both L1 and L2 frequencies. Mean values w.r.t. the tide gauge series have been removed, and to improve the readability of the figure the time series were shifted with offsets of $30 \mathrm{~cm}$. Lower plot: Wind speed measurements from the weather station at the Onsala Space Observatory.

series appear to be rather noisy with a scattering that amounts to a significant fraction of the daily variation of sea level. The SMHI tide gauge has a sampling rate of only one minute which means that the two instruments can only be compared at the epoch of the radar tide gauge. As the sampling of the SNR and single difference solution is irregular and larger than those of the GLONASS-R results, tide gauge data were interpolated to match the epochs of the data from the GNSS-R system based on commercial GNSS receivers. Figure 5 depicts the root mean square error (RMSE) of the individual time series w.r.t. the SMHI tide gauge after removing a mean offset from each series. The SNR solutions agree with the official tide gauge measurements with a standard deviation between 8 and $10 \mathrm{~cm}$, and the single difference solutions from GPS and GLONASS phases reveal RMSE values between 5 and $6 \mathrm{~cm}$. These values are slightly larger than those reported in [10], though one has to consider that during the whole period of the experiment relatively strong winds were present at the OSO. Both GLONASS-R solutions, the low-pass filtered B-spline results and the inverted interferometer phases, show smaller RMSE w.r.t. the SMHI tide gauge. The B-spline results have a RMSE below $2 \mathrm{~cm}$ and the high frequency measurements agree with the tide gauge data with a standard deviation slightly larger than $3 \mathrm{~cm}$. The better agreement of the GLONASS-R results can be attributed to the way how relative phases between direct and reflected signals are obtained. The GNSS-R system 


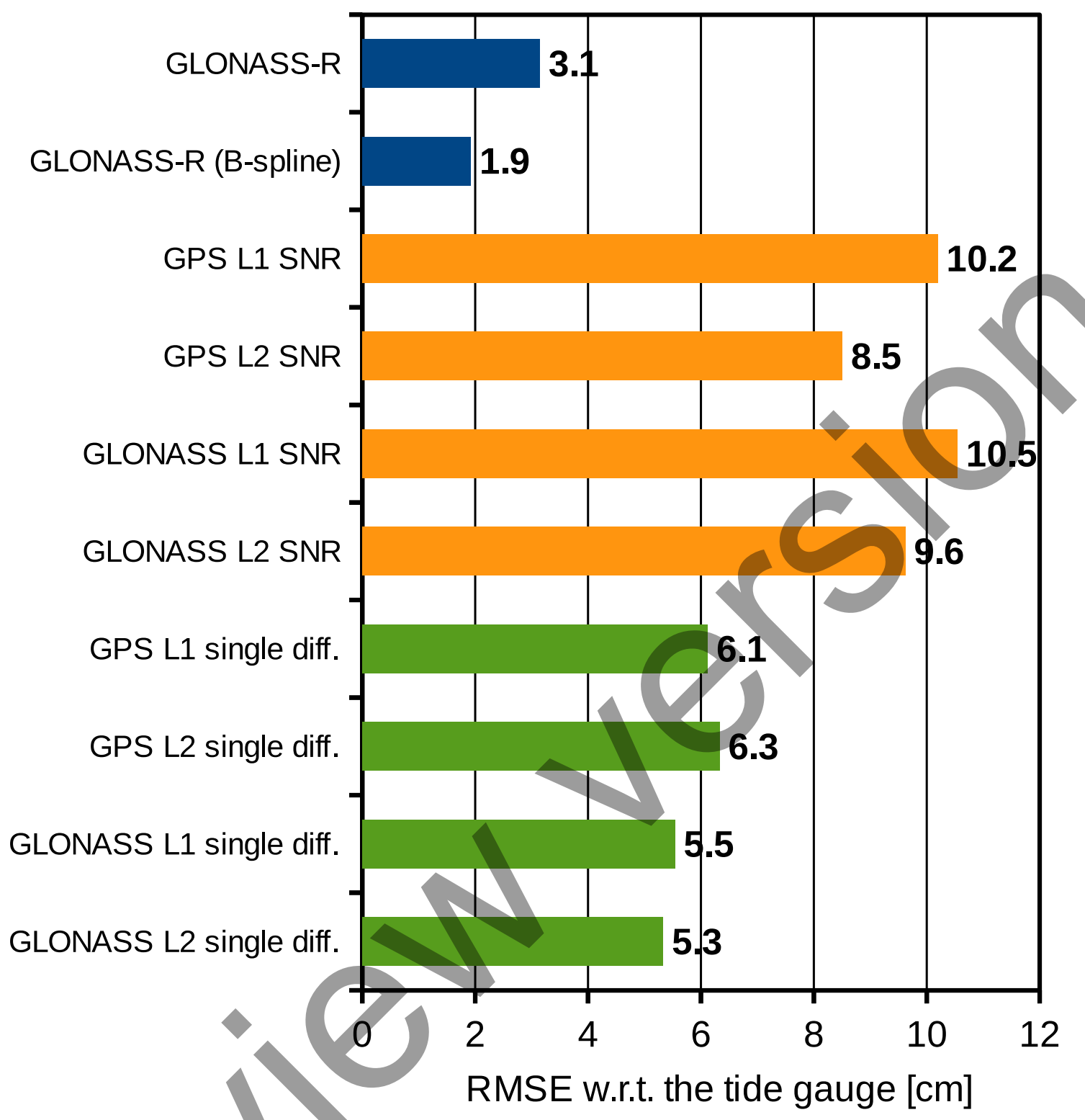

Fig. 5. Root mean-square errors (RMSE) of the individual time series w.r.t. the SMHI tide gauge measurements. Note: As offsets were removed before computing RMSE, the results can also be interpreted as standard deviations of the time series differences.

based on commercial receivers tracks RHCP and LHCP signals independently and cycle slips, which are more likely in the weaker and more disturbed LHCP channel, are occurring more often and thus complicate and weaken the single difference solutions. On the other side, the GLONASS-R system which measures the interferometer phase directly might be also affected by weak signals due to rough surface conditions, but both the long integration time of 0.67 seconds and the model based phase unwrapping approach avoid that phase data from low SNR trackings impact the inversion process strongly. In order to check whether GLONASS-R measurements vary in dependence of the wind speed, i.e. agree less with tide gauge data when the sea surface roughness increases, standard deviations between the two time series were computed for different wind speed categories. 


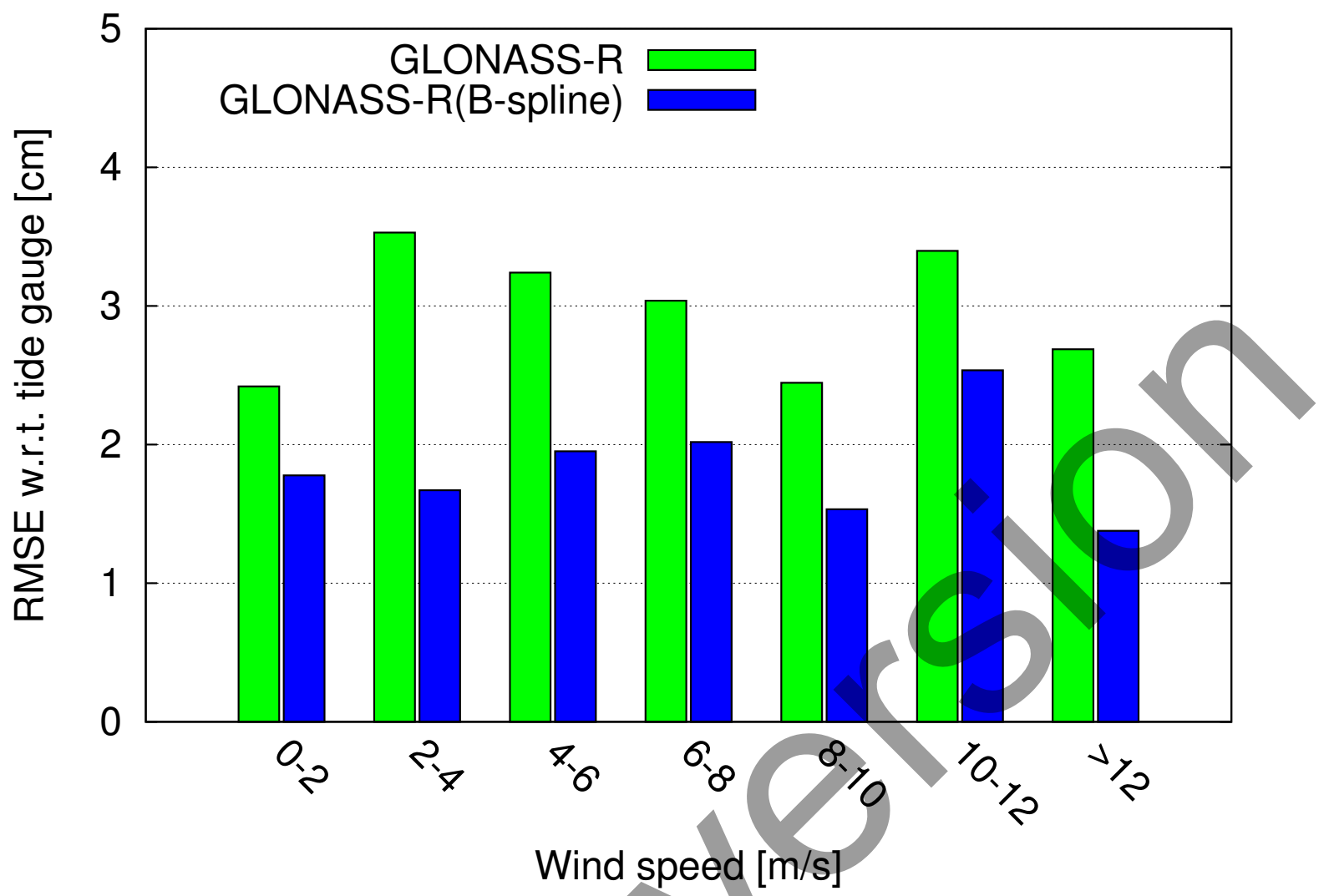

Fig. 6. Root-mean-square error (RMSE) of the two GLONASS-R time series w.r.t. the SMHI tide gauge readings, grouped for different wind speeds.

These results are depicted in Fig. 6 and it is confirmed that the GLONASS-R system provides data with the same precision independent of the wind speed, respectively the resulting sea surface roughness. This differs from the findings reported in [14] where it was concluded that higher wind speeds lead to less precise sea level retrievals from the GLONASS-R system. However, the system discussed in Sec. II differs from the earlier concept in many aspects, whereas the fact that coherent integration has been replaced by the usage of very large FFTs is expected to be the main contributor in making the system more robust against weak signals corresponding to reflections from rough sea surfaces.

Haying studied how well the individual time series agree w.r.t. the SMHI tide gauge measurements, the question remains whether and to which extent the GLONASS-R is able to sense sea level signals.

\section{B. Spectral analysis}

During the whole period of the experiment only the GLONASS-R time series have a regular sampling and do not contain data gaps. Tide gauge data are available with a regular but coarser temporal resolution, though the data gap around June 15 splits the time series in two parts. In order to avoid bridging a gap of more than two days, only the period between June 19, 2015 and the end of the experiment, i.e. July 3rd is considered in the following. The sampling rate of $0.67 \mathrm{~s}$ for the GLONASS-R solution and $60 \mathrm{~s}$ for SMHI tide gauge observations define 


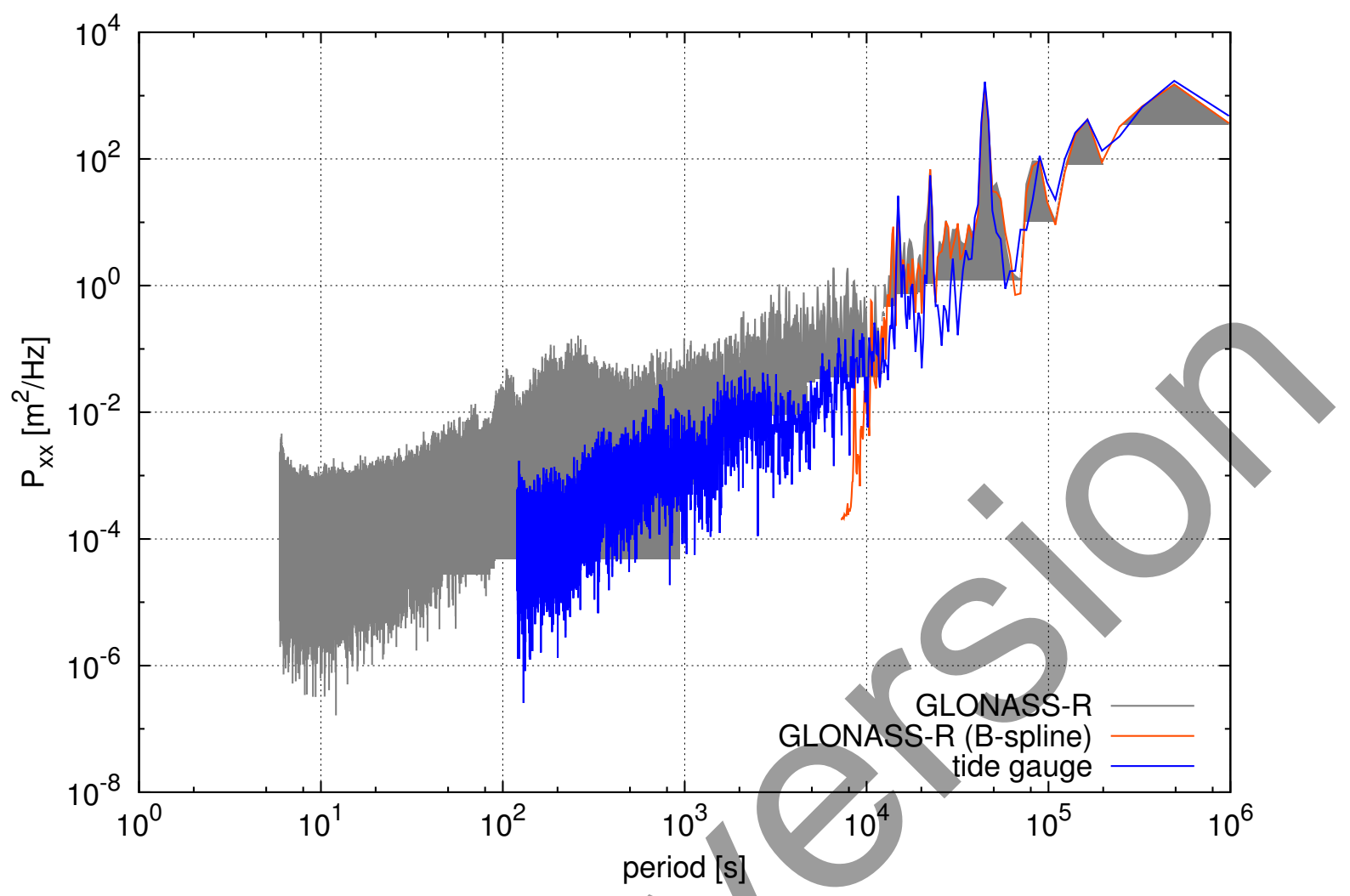

Fig. 7. Power spectral density (PSD) estimates from the GLONASS-R time series and the tide gauge data for the period June, 19 - July, 3 , 2015.

the Nyquist frequency for any spectral analysis. B-spline low-pass filtered results are available at any arbitrary resolution as the sea surface is described by a continuous function model (cf. Eq. 5). However, periods less than the temporal spacing of the B-spline nodes should not be considered due to the strong effect of the low-pass filtering process. In the following, the B-spline solution has been evaluated with a temporal spacing equal to the SMHI tide gauge, i.e. $60 \mathrm{~s}$, but spectral components with periods less than $7200 \mathrm{~s}$ were removed in order to preserve only the meaningful parts of the spectrum. Power spectral density estimates were computed for all three time-series using Welch's method [21]. Figure 7 depicts these spectra in dependency of the signal period. It is clearly visible that both GLONASS-R results and the tide gauge measurement have identical spectral components for periods longer than 3 hours (10800 s). Distinct peaks, in particular those corresponding to daily and half-daily signals can be assigned to ocean and atmosphere tides. The latter one might be unexpected, but given that the half open bay at the OSO is causing sea surface to correlate strongly with air pressure changes it is not a surprise that not only ocean but also atmospheric tides contribute to the pattern of sea level at various time scales.

For periods shorter than 3 hours, GLONASS-R and the SMHI tide gauge time series seem to contain no clear peaks. However, one notices that the GLONASS-R spectrum has a slope which is less steep than those of the tide gauge. This might be attributed to the fact that the radar tide gauge is hosted inside a stilling well which is connected to the open water by a couple of underwater pipes. Thus, sea-level as sensed by the GLONASS-R system is strictly 
speaking a snapshot of the sea surface corresponding to the average of the satellite's footprints/Fresnel zone as seen from the LHCP antenna. On the other side, the water level inside the well is determined by the integral pressure of the sea water next to the tide gauge. Thus, the stilling well acts as a low pass filter, in particular it does not map outside sea surface conditions as stimulated by wind to the inside where the radar is mounted. Thus, it is obvious that the spectra start to differ for wavelengths which are $r$ elated to winds and local conditions.

In addition, it appears that the GLONASS-R spectrum flattens out for periods shorter than $100 \mathrm{~s}$ which indicates that the instruments noise level starts to dominate the spectral content for such short periods. Thus, one can state that the GLONASS-R system is capable to detect exactly the same tidal periods as measured by the conventional tide gauge. In general, one needs to take into account that for the interpretation of shorter period signals one should recall that any GNSS-R system represents the average sea surface heights of all satellite foot-prints, whereas an in-situ or stilling well hosted tide gauges basically monitor an integral of the water level next to the tide gauge.

\section{CONCLUSIONS AND OUTLOOK}

The GLONASS-R concept, first presented in [14], has been developed further by making use of software-defined radio off-the-shelf components and optimizing the signal and post processing strategies. Several test runs, including a long term experiment over three weeks (cf. Sec. III) confirm the potential of the system. Signal processing on the GPU proves to be a suitable solution in order to handle the large sampling rates and process up to 12 satellites in real-time. Since all components are commercially available at rather moderate prices, GLONASS-R systems can be duplicated fairly easy and tested on other sites. Looking through the list of necessary components it appears that the high-end geodetic GNSS antennas would amount for the largest fraction of the total cost of a GLONASS-R system. Such antennas were used as they existed already at the OSO GNSS-R site. If one wants to duplicate a complete GLONASS-R system much cheaper GLONASS capable antennas are available as commercial products, and even dedicated LHCP antennas are sold for prices less than those of standard gaming GPU cards.

The long term test, discussed in Sec. III has demonstrated that the system can produce interferometric phases continuously with sampling rates of higher than $1 \mathrm{~Hz}$. The updated signal processing concept of using large FFTs instead of coherent integration of shorter pieces of data has proven to be very successful. In particular, during periods of rougher sea surface conditions, the new cross-correlation approach still provides meaningful interferometer phases which do not cause artifacts in the inverted reflector height products. This goes along with the new post-processing scheme, in which phase unwrapping is carried out by a boot-strapping approach using an empirical model of the reflector during the passage of one satellite. Although the three-step approach described in Sec. II-C has proven that it is capable of providing precise results with high temporal resolution, a direct inversion of the interferometer phases is expected be even more robust. However, this requires that Eq. 1, which contains the discontinuous modulo operator, can be used by a parameter estimation method. First efforts in this direction have been made e.g. by [22][24]. Tests with such proposed algorithms have been carried out but results indicated that the obtained sea surface height measurements contain more artifacts than those coming from the three-step approach. The main reason for this is found to be in the fact that there is no straightforward solution that allows to fit model parameters according to Eq. 1. More theoretical work in this direction and the usage of mathematical algorithms which have been designed 
for similar problems are expected to overcome this drawback and improve the GLONASS-R products further.

As shown in Sec. III, GLONASS-R agrees better with measurements of a co-located conventional tide gauge, than GNSS-R data from commercial GPS receivers do. The SNR method, using only data from the upward looking RHCP antenna, provides results even during period of rough sea surface conditions, but the precision of these temporally sparse sea surface measurements is only slightly better than about $10 \mathrm{~cm}$. On the other side, single-difference phase solutions, have the potential to be at least as precise as the GLONASS-R results (cf. [10]), however the fact that commercial GNSS receivers are dealing with reflected signals by using standard GNSS tracking algorithms it happens rather often that cycle slips or large data gaps occur during periods when the reflector surface is rough. Thus, the single difference method fails or produces time-series with rather low precision during such periods. The GLONASS-R system, which produces interferometer phases for all conditions, allows to deal with the circumstances of the reflector's physical properties, by considering the SNR values obtained from the software receiver. Moreover, as phases are obtained within the range $[-\pi, \pi]$ raw data are free of cycle slips and the boot-strapping phase unwrapping method described in Sec. II-C1 avoids that cycle slips are introduced at a later stage. Thus, it is not a surprise that the inverted GLONASS-R results agree with the tide gauge measurements on the level of 2 and $3 \mathrm{~cm}$, for B-spline and high frequency r eflector height measurements, respectively.

Further tests and validation campaign with ground based GNSS-R systems from different research groups are anticipated for the near future. In addition, tests with the GLONASS-R system on other locations are being considered as well.

In general, the concept presented here has to be seen under the consideration of GLONASS modernization plans which include the possible use of the CDMA technique instead of the current FMDA concept. Thus, further testing and development depend also on the availability of these FDMA signals.

This research has been conducted with support from "Stiftelsen Lars Hiertas Minne" (FO2014-0663) and "Adlerbertska forskningsstiftelsen". The commercial GNSS-equipment (receivers, antennas) were purchased via the Leica Geosystems ATHENA program. The authors also thank teams from the workshop and the electronics laboratory at the Onsala Space Observatory for preparation of the infrastructure at the test site. We gratefully acknowledge the support of NVIDIA Corporation with the donation of the Tesla K40 used for this research.

\section{REFERENCES}

[1] X. Collilieux and G. Wöppelmann, Global sea-level rise and its relation to the terrestrial reference frame, Journal of Geodesy, 85(1), 9-22, 2011.

[2] K. D. Anderson, Determination of Water Level and Tides Using Interferometric Observations of GPS Signals, J. Atmos. Oceanic Technol., 17, 1118-1127, 2000.

[3] K. M. Larson, R. D. Ray, F. D. Nievinski and J. T. Freymueller, The Accidental Tide Gauge: A GPS Reflection Case Study From Kachemak Bay, Alaska, Geoscience and Remote Sensing Letters, IEEE , 10, 5, 1200-1204, 2013.

[4] K. M. Larson, J. Löfgren and R. Haas, Coastal Sea Level Measurements Using A Single Geodetic GPS Receiver, Adv. Space Res., 51(8), 1301-1310, 2013.

[5] Y. Nakashima and K. Heki, GPS tide gauges using multipath signatures, J. Geod. Soc. Japan, 59, 157-162, 2013. 
[6] J. S. Löfgren, R. Haas and H. G. Scherneck, Sea level time series and ocean tide analysis from multipath signals at five GPS sites in different parts of the world, Journal of Geodynamics, 80, 66-80, 2014.

[7] J. S. Löfgren, R. Haas and J.M. Johansson, Monitoring coastal sea level using reflected GNSS signals, Adv. Space Res., 47, 2,213-220, 2011.

[8] R. Treuhaft, S. Lowe, C. Zuffada, and Y. Chao, Two-centimeter GPS altimetry over Crater Lake, Geophys. Res. Lett., 28,23, 4343-4346, 2001.

[9] J. S. Löfgren, R. Haas, H.-G. Scherneck and M.S. Bos, Three months of local sea level derived from reflected GNSS signals, Radio Sci., 46, 2011), p. RS0C05

[10] J. S. Löfgren and R. Haas, Sea level measurements using multi-frequency GPS and GLONASS observations, EURASIP Journal on Advances in Signal Processing, 1, 2014.

[11] M. Skolnik, Radar Handbook, Third Edition, McGraw-Hill, 1328p., ISBN 0071485473, 2008.

[12] N. J. Willis and H. Griffiths, Advances in Bistatic Radar, SciTech Publishing, 493p.,ISBN 1891121480, 2007.

[13] A. Rius, O. Nogues-Correig, S. Ribo, E. Cardellach, S. Oliveras, E. Valencia, H. Park, J. Tarongi, A. Camps, H. Marel, R. Bree, B. Altena and M. Martin-Neira, Altimetry with GNSS-R interferometry: first proof of concept experiment, GPS Solutions, 16(2), 231-241, 2012.

[14] T. Hobiger, R. Haas and J. S. Löfgren, GLONASS-R: GNSS reflectometry with a Frequency Division Multiple Access-based satellite navigation system, Radio Sci., 49, 271-282, 2014.

[15] J. H. Reed, Software Radio: A Modern Approach to Radio Engineering, Prentice Hall PTR, 592p., ISBN 0130811580, 2002.

[16] N. Guo, S. Backen and D. Akos, The Universal Software Radio Peripheral as RF Front-End, GPS World, March 2013, 36-41, 2013.

[17] CelesTrak (2013), NORAD Two-Line Element Sets, http://www.celestrak.com/.

[18] R. E. Ladner and M. J. Fischer,Parallel Prefix Computation,J. ACM, 27(4), 831-838, 1980.

[19] K. R. Koch, Parameter Estimation and Hypothesis Testing in Linear Models, Springer, Berlin, 334p., ISBN 9783540652571, 1999.

[20] J. W. Eaton, D. Bateman and S. Hauberg, GNU Octave version 3.0.1 manual: a high-level interactive language for numerical computations, CreateSpace Independent Publishing Platform, ISBN 1441413006, http://www.gnu.org/software/octave/doc/interpreter/, 2009.

[21] P. D. Welch, The Use of Fast Fourier Transform for the Estimation of Power Spectra: A Method Based on Time Averaging Over Short, Modified Periodograms, IEEE Transactions on Audio Electroacoustics, AU-15, 70-73, 1967.

[22] J. C. Kucwaj, G. Stienne, S. Reboul, J. B. Choquel and M. Benjelloun, Circular multiple change-points estimation applied to the GPS-L2C phase signal, 17th International Conference on Information Fusion (FUSION), 1-6, 2014.

[23] G. Stienne, S. Reboul, J. B. Choquel and M.Benjelloun, Cycle slip detection and repair with a circular on-line change-point detector, Signal Processing, 100, 51-63, DOI;10.1016/j.sigpro.2014.01.003 2014.

[24] G. Stienne, S. Reboul, M. Azmani, J. B. Choquel and M. Benjelloun, A multi-temporal multi-sensor circular fusion filter, Information Fusion, 18, July 2014, 86-100, DOI:10.1016/j.inffus.2013.05.012., 2014.

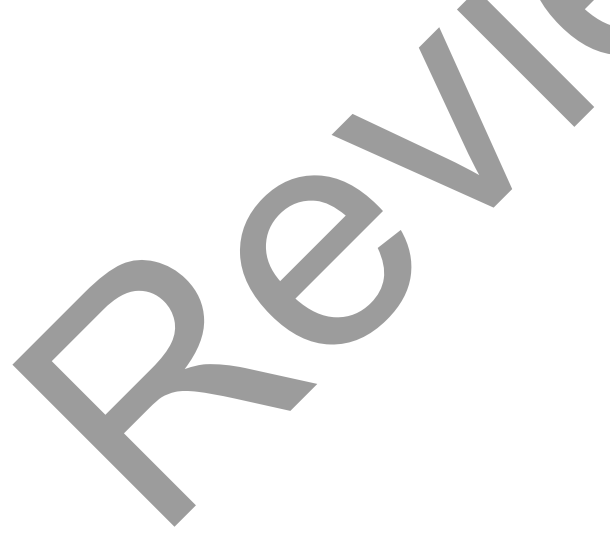

Thomas Hobiger Thomas Hobiger is Associate Professor for Space Geodesy at Chalmers University of Technology. He plays an active role in the development of the next-generation space-geodetic systems (in particular VLBI and GNSS) and processing tools for such techniques. $\mathrm{He}$ is interested in VLBI, GNSS, Earth rotation, sea-level change and GNSS-R, software-defined radio, reference frames, high-performance computing, propagation of radio waves, and time and frequency transfer. Moreover, he is an IAG Fellow and has received the Tsuboi Award 2010 of the Japanese Geodetic Society, the EPS Award 2010 and the EGU Outstanding Young Scientist Award 2011. Thomas currently serves on the editorial board of the Journal of Geodesy and is actively involved in several working groups of scientific communities. 
Rüdiger Haas Rüdiger Haas is Professor for Space Geodesy at Chalmers University of Technology and group leader for the research group on Space Geodesy and Geodynamics. He is responsible for the geodetic VLBI activities at the Onsala Space Observatory. Rüdiger's reseach work deals with space geodesy and global geophysical phenomena, as e.g. Earth rotation, global reference frames, change in atmospheric water vapour, sea level measurements. Rüdiger is the scientific leader of the Onsala Twin Telescope project, chair of the European VLBI Group for Geodesy and Astrometry (EVGA) and member of both the directing boards of the International VLBI Service for Geodesy and Astrometry (IVS) and the International Earth Rotation and Reference Frames Service (IERS).

Johan Löfgren Johan Löfgren holds a PhD degree from Chalmers University of Technology. He is interested in research related to GNSS (Global Navigation Satellite Systems, e.g., GPS) and its applications. Johan's primary research areas are reflected GNSS signals, multipath, and positioning with GNSS. Foremost, Johan's research is directed towards local sea level measurements with GNSS signals. Johan's research is carried out both with the GNSS tide gauge at the Onsala Space Observatory and with data from GNSS stations all around the world. 